\title{
Syntactic Unification Operations Are Reflected in Oscillatory Dynamics during On-line Sentence Comprehension
}

\author{
Marcel Bastiaansen $^{1,2}$, Lilla Magyari ${ }^{1}$, and Peter Hagoort ${ }^{1,2}$
}

\begin{abstract}
There is growing evidence suggesting that synchronization changes in the oscillatory neuronal dynamics in the EEG or MEG reflect the transient coupling and uncoupling of functional networks related to different aspects of language comprehension. In this work, we examine how sentence-level syntactic unification operations are reflected in the oscillatory dynamics of the MEG. Participants read sentences that were either correct, contained a word category violation, or were constituted of random word sequences devoid of syntactic structure. A time-frequency analysis of MEG power changes revealed three types of effects. The first type of effect was related to the detection of a (word category) violation in a syntactically structured sentence, and was found
\end{abstract}

\section{INTRODUCTION}

The cognitive architecture of language comprehension is considered to consist of at least two different cognitive operations (Hagoort, 2005; Jackendoff, 2002). Memory retrieval operations subserve the retrieval of phonological, syntactic, and semantic properties of words from long-term memory. Unification operations (which are also considered to take place at the phonologic, syntactic, and semantic levels) combine the information about individual words into an overall representation of the multiword utterance. Hemodynamic neuroimaging techniques have consistently reported activations in left temporal areas during memory retrieval operations, whereas unification operations have been shown to elicit activation in left inferior frontal areas (for reviews, see Hagoort, 2005; Indefrey \& Cutler, 2005; Indefrey, 2004; Bookheimer, 2002). Thus, by and large, the brain appears to differentiate between memory and unification operations.

However, a few decades of cognitive neuroscience research have made it very clear that cognitive functions rely on the connectivity patterns within large-scale neuronal networks, rather than on strictly localized processes. Indeed, over the last 15 years, evidence has accumulated that neuronal synchronization is related to the coupling

\footnotetext{
${ }^{1}$ Max Planck Institute for Psycholinguistics, Nijmegen, the Netherlands, ${ }^{2}$ Donders Institute for Brain, Cognition, and Behavior, Centre for Cognitive Neuroimaging, Nijmegen, the Netherlands
}

in the alpha and gamma frequency bands. A second type of effect was maximally sensitive to the syntactic manipulations: A linear increase in beta power across the sentence was present for correct sentences, was disrupted upon the occurrence of a word category violation, and was absent in syntactically unstructured random word sequences. We therefore relate this effect to syntactic unification operations. Thirdly, we observed a linear increase in theta power across the sentence for all syntactically structured sentences. The effects are tentatively related to the building of a working memory trace of the linguistic input. In conclusion, the data seem to suggest that syntactic unification is reflected by neuronal synchronization in the lower-beta frequency band.

and uncoupling of functional networks in the brain (see, e.g., Varela, Lachaux, Rodriguez, \& Martinerie, 2001; Pfurtscheller \& Lopes da Silva, 1999b; Singer, 1993, 1999; Pfurtscheller \& Neuper, 1997; Pfurtscheller \& Berghold, 1989, and many more). The idea is that synchronous, repetitive firing of neurons facilitates the activation of functional networks because it increases the probability that neurons entrain one another in synchronous firing (e.g., Konig \& Schillen, 1991). In addition, elements pertaining to one and the same functional network are identifiable as such by virtue of the fact that they fire synchronously, at a given frequency. This frequency specificity allows one and the same neuron (or neuronal pool) to participate at different times in different representations. Hence, synchronous oscillations in a wide range of frequencies are considered to play a crucial role in linking areas that are part of one and the same functional network. Importantly, in addition to recruiting all the relevant network elements, oscillatory neuronal synchrony would also serve to bind together the information that is represented in the different elements, as was elegantly demonstrated in a seminal paper by Gray, Konig, Engel, and Singer (1989).

Recent accounts of language comprehension therefore emphasize the importance of dynamic interactions between different parts of the brain during language comprehension, and stress the distributed nature of the brain's language network (e.g., Bastiaansen \& Hagoort, 
2006; Salmelin \& Kujala, 2006; Pulvermueller, 1999). In this context, it should be noted that over the past few decades there has been a recurrent debate about whether oscillatory neuronal ensembles can handle the hierarchical structure of language (e.g., Pulvermueller, 2002; Hummel \& Holyoak, 1997). Recently there have been successful attempts at modeling hierarchical categories (Huyck, 2006), and natural-language parsing, by means of neural networks consisting of distributed cell assemblies.

Therefore, it is not surprising that in recent years there have been an increasing number of studies aimed at relating changes in neuronal synchronization (as expressed by event-related changes in scalp-recorded EEG/MEG power and/or coherence) to different aspects of language comprehension (see, e.g., Bastiaansen \& Hagoort, 2006; Weiss \& Mueller, 2003 for reviews). In an attempt to relate these studies to the cognitive architecture of language comprehension (Bastiaansen \& Hagoort, 2006), we have noted that a number of studies point toward an important role for oscillatory synchrony in the theta frequency range in memory retrieval operations (roughly 4-7 Hz; Bastiaansen, Oostenveld, Jensen, \& Hagoort, 2008; Bastiaansen, Van der Linden, ter Keurs, Dijkstra, \& Hagoort, 2005; Bastiaansen, Van Berkum, \& Hagoort, 2002a), which fits well with the more general relationship between theta-band synchronization and memory retrieval processes (reviewed in Bastiaansen \& Hagoort, 2003; Klimesch, 1999). Other data additionally suggest a possible involvement of alpha frequency range (around $10 \mathrm{~Hz}$ ) synchronization in lexical retrieval (Rohm, Klimesch, Haider, \& Doppelmayr, 2001; Klimesch, Doppelmayr, Pachinger, \& Ripper, 1997; Klimesch, Doppelmayr, Pachinger, \& Russegger, 1997). In contrast, there is increasing evidence that sentence-level semantic unification operations are accompanied by increases in oscillatory synchrony in the gamma frequency range (roughly 30-80 Hz; Hald, Bastiaansen, \& Hagoort, 2006; Hagoort, Hald, Bastiaansen, \& Petersson, 2004; Van Berkum, Zwitserlood, Bastiaansen, Brown, \& Hagoort, 2004; Braeutigam, Bailey, \& Swithenby, 2001). From these studies it appears that normal semantic unification goes together with a modest increase in gamma-band synchronization, which is disrupted when semantic unification becomes problematic.

With respect to unification operations at the syntactic level, the picture is less clear. To date, only two studies have explicitly addressed the oscillatory neuronal dynamics during syntactic unification. The first study (Bastiaansen, Van Berkum, \& Hagoort, 2002b) investigated power changes in the alpha and theta frequency ranges of the EEG during the processing of sentences containing different types of syntactic violations (number agreement violations and grammatical gender violations). Both types of violations elicited a phasic theta power increase at frontal electrodes, albeit with a different lateralization. The second study (Weiss et al., 2005) compared EEG coherence changes elicited by syntactically complex sentences containing object-relative clauses versus the much simpler (and canonical) subject-relatives. The authors showed that object-relatives elicit higher coherence in the theta and gamma ranges during the processing of the relative clause, and in the theta and lower-beta (13-18 Hz) ranges during the postrelative clause interval. They related the theta-band effects to increased working memory demands, the gamma-band effects to higher attentional processing, and the beta-band effects to more extensive semanticpragmatic analysis. However, elsewhere (Weiss \& Mueller, 2003) the authors argue that the beta and gamma effects may be related to syntactic and semantic analysis, a view that better converges with our own observations (as far as gamma and semantic unification is concerned). To summarize, the available data concerning oscillatory neuronal synchrony and syntactic unification do not yield a very clear picture. This work therefore primarily aims at further specifying the oscillatory dynamics during syntactic unification operations.

A second aim of the article is to provide a description of synchronization changes across entire sentences. The reason for this is the following. The aforementioned notion of binding by synchrony (Fries, 2005; Varela et al., 2001; Singer, 1999; Gray et al., 1989) was first introduced in the context of the processing of sensory (most notably visual) information. In sensory systems, different aspects of the sensory input (e.g., different visual features such as color and shape) impinge more or less simultaneously on the relevant cortical areas. During language comprehension, however, the brain has to deal with sequential input which is spread out over relatively long time stretches of up to several seconds, or even longer (e.g., in the case of integrating incoming words in the discourse context, see, for instance, Van Berkum, Koornneef, Otten, \& Nieuwland, 2007; Nieuwland \& Van Berkum, 2006). This implies, in the context of reading sentences, that a working memory trace of the linguistic input has to be gradually built up across the sentence. The unification (binding) of syntactic and semantic information then has to take place with preceding context, rather than with simultaneously impinging stimulus properties (see, e.g., Gibson, 1998 for a theoretical account of the dissociation between syntactic unification and working memory during language comprehension). Previously, we have found that theta power gradually increases across the sentence, which may be related to the formation of a verbal working memory trace (Bastiaansen et al., 2002a). However, in that study, we only computed alpha and theta power changes. In addition, we only considered syntactically correct sentences. Therefore, syntactic and semantic unification operations operate in parallel with, and probably interact with, the formation of a working memory trace Thus, in the previous study (Bastiaansen et al., 2002a), it was not possible to dissociate syntactic structure-building operations and semantic unification across the sentence from the formation of a working memory trace.

To address the above issues, we recorded the MEG of subjects while they read correct sentences, sentences 
containing a simple syntactic violation (a relatively mild word category violation, in which a critical noun was replaced with a verb by adding a verbal inflection to the stem of the noun), and random word sequences that were devoid of syntactic structure (created by pseudorandomizing the order of the words of the correct sentences). In addition to the standard evoked-fields analysis, we computed time-frequency (TF) representations of the evolution of power, both time-locked to the onset of the critical word (CW; i.e., the word at which the violation occurs in the word category violation condition) and across the entire sentence (i.e., time-locked to the sentence-initial word) in a wide range of frequencies, from 2 to $100 \mathrm{~Hz}$.

Although there are no clear hypotheses about the oscillatory neuronal dynamics of syntactic processing, let us tentatively assume (based on the faint evidence that can be found in the study by Weiss et al., 2005) that, analogous to the gamma results obtained with semantic violation manipulations (e.g., Hald et al., 2006; Hagoort et al., 2004), oscillations in the beta frequency range are functionally related to syntactic unification operations. This assumption would lead us to predict the following pattern of results. In the analysis of the CW-locked data, we hypothesize a decrease in beta power in response to the presentation of the local word category violations relative to the corresponding word in the correct sentence. Such a drop in beta power would reflect a failing syntactic unification process. In the analysis of the sentence-onset-locked data, we predict to observe a beta power increase across the sentence for syntactically correct sentences, and for sentences containing word category violations, but only up to the violating word. For the random word sequences, which do not contain any syntactic structure, we expect such a beta power increase across the sentence to be absent because syntactic structure-building cannot take place in this condition. Finally, in the sentence-onset-locked analysis, we additionally expect to replicate the theta power increase across the sentence we previously reported. If this effect indeed reflects the gradual building of a working memory trace, as we have argued elsewhere (Bastiaansen et al., 2002a), we would expect it to be less sensitive to the syntactic manipulations than the putative effects in the beta band.

\section{METHODS}

\section{Participants}

Twenty-three native speakers of Dutch (9 men and 14 women) participated in the experiment after having given informed consent. None had neurological impairments, experienced neurological trauma, or had used neuroleptics. The participants were paid a small fee for their participation. Three participants' data were discarded from further analysis due to poor data quality. From the remaining 20 participants, 18 (11 women, 7 men; age range $=18-45$ years) were included in the final anal- ysis so as to obtain an equal number of participants (6) in each of the three versions of the experiment (see Stimulus Material and Experimental Design section).

\section{Stimulus Material and Experimental Design}

The experimental stimulus material consisted of 180 triplets of Dutch sentences. A sentence triplet contained the following three conditions (see Table 1 for examples of the stimulus materials). The correct sentence (COR) condition consisted of grammatically correct Dutch sentences that varied in length from 6 to 10 words. The second condition was a word category violation condition, in which a word category violation was introduced at the noun following the main verb. The position of this CW varied from the third to the seventh position. The word category violation was achieved by adding a verbal inflection to the stem of the corresponding noun in the correct version, which clearly signaled the verbal category (e.g., jager $\rightarrow$ jagen; bunter $\rightarrow$ "to bunt"; see also Table 1$)$. In the third condition, the random word order (RDM) condition, all the words of the correct sentence were presented without modification but in a random order (Table 1C). Only the CW was kept at the same position as in the correct sentence condition. After randomizing word order, it was verified that the resulting sentence did not longer contain any meaningful syntactic structure. In the rare cases where the randomization procedure did result in a possible syntactic structure, words were manually displaced so as to undo this structure.

In addition to the 180 experimental sentences, the stimulus material also contained 240 filler sentences, which contained either object-relative or subject-relative clauses. These filler sentences were constructed for another experimental manipulation, and are not analyzed in the present study.

We wanted to avoid sentence repetition effects. Therefore, we made sure subjects saw only one sentence of each experimental triplet of sentences by creating three different versions (experimental lists) of the experiment, in which sentences within each triplet were pseudorandomly assigned to one version. Thus, the full extent of the experimental materials was distributed across the three different versions. It is therefore essential that these versions be presented an equal number of times

Table 1. Example Stimuli in Each Condition

\begin{tabular}{ll}
\hline A: COR & Janneke kreeg de zegen bij de rivier. \\
& (Janneke got the blessing at the river). \\
B: CAT & Janneke kreeg de zegenen bij de rivier. \\
& (Janneke got the "to bless" at the river). \\
C: RDM & De de Janneke zegen kreeg rivier bij. \\
& (The the Janneke blessing got river at). \\
\hline
\end{tabular}

Literal English translation in italics. 
(i.e., to have an equal number of subjects for each version). The experimental lists were derived from a single randomization, created by pseudorandomly mixing the 180 critical items with the 240 filler items, such that the largest sequence of consecutive critical trials was four. The experimental lists were split up in four blocks of 105 trials.

Each trial started with a blank screen for $300 \mathrm{msec}$, which was followed by a word-by-word presentation of the sentences. The words were presented in white fonts on a gray background, in the middle of the screen. Each word was on screen for $300 \mathrm{msec}$, followed by a 300-msec long blank screen. Stimulus onset asynchrony was $600 \mathrm{msec}$. After the offset of the last word in the sentence and before the onset of the first word of the next sentence, an asterisk was presented for $2600 \mathrm{msec}$, giving time to the subjects to blink. Multiple-choice questions testing for reading comprehension were inserted at random intervals at the end of the filler sentences (600 msec after onset of the sentence-final word) in $10 \%$ of the cases.

\section{Procedure}

Before the MEG measurement took place, subjects were asked to read information about the MEG measurement procedure and the instructions about the experiment. They then filled out an informed consent form and the Dutch version of the Edinburgh Handedness Inventory.

The measurement took place in four blocks of approximately $20 \mathrm{~min}$. Subjects were allowed to leave the magnetically shielded room in between blocks. Head position was measured before and after each block, and the average of both head position measurements was used to determine the position of the sensors with respect to the head. Subjects were asked to minimize their head movements during the blocks and to refrain from blinking during reading the sentences. In order to familiarize the subjects with the procedure, a practice block of four sentences and three questions related to the sentences preceded the first experimental block. The entire session, including subject preparation, instructions, practice block, and debriefing, took approximately $80 \mathrm{~min}$.

\section{MEG Recordings}

MEG recordings took place in a dimly illuminated, soundattenuating, magnetically shielded room. MEG signals were recorded with a 151-channel whole-head MEG system (VSF/CTF Systems, Port Coquitlam, Canada). In addition, EOG was recorded bipolarly to later discard trials contaminated by eye movements and blinks. Horizontal EOG was recorded with electrodes placed on the outer canthi. For the vertical EOG, a sub- to supraorbital montage was used. A ground electrode was placed on the subject's left arm. MEG and EOG signals were amplified from DC to $300 \mathrm{~Hz}$, and recorded continuously with a sampling rate of $1200 \mathrm{~Hz}$.
Three head localization coils were placed on anatomical landmarks of the head (at the nasion and in the left and right ear canal) in order to monitor the head position with respect to the MEG helmet. The head position was determined before and after each of the four experimental blocks.

All stimuli were presented to the subjects on a mirror by a back-projection system where an LCD projector was placed outside the magnetically shielded room in order to avoid interfering electrical apparatus.

\section{Data Preprocessing}

The data analysis was performed with the Fieldtrip software package, a Matlab-based toolbox dedicated to the analysis of electromagnetic and electrophysiological data (www.ru.nl/fcdonders/fieldtrip/). First, power line fluctuations were removed from the continuous MEG recordings by estimating and subtracting the 50-, 100- and 150-Hz components in the data, using a discrete Fourier transform on 10-sec data segments surrounding the data epochs of interest. Next, trials ranging from $2500 \mathrm{msec}$ before the onset of the first word in a sentence to $2500 \mathrm{msec}$ after sentence offset were segmented. Trials containing artifacts were excluded from further analysis (38\% in total). The amount of excluded trials was approximately equal across conditions $[F(2,34)=1.65, p=.207]$, yielding an average of approximately 37 artifact-free trials in each condition. The relatively high proportion of rejections was mainly due to the fact that the epochs were very long (ranging from $8900 \mathrm{msec}$ for the shortest sentences to $11,300 \mathrm{msec}$ for the longest sentences), and therefore contained a substantial amount of eye-movement artifacts.

Next, because we were interested in a topographical analysis of the data, we computed the planar gradients of the axial gradiometer-recorded data by taking, for each sensor, the average of the absolute values of the first spatial derivatives in two orthogonal directions. The resulting data are equivalent to data recorded with MEG systems with planar gradiometers, which pick up maximum signal above the source. However, one should keep in mind that in the resulting planar gradients noise is spread across sensors: In the axial data, each sensor has its own noise, whereas in the planar transform each sensor has a weighted average of its own noise and that of its neighbors. In addition, at the edges of the sensor array, there are less "neighbors" for each sensor to base the spatial derivative on, so the estimates are somewhat less reliable there. Despite these relative disadvantages of the axial-to-planar conversion, scalp topographies of power changes dramatically improve when computed on planar transformations as compared to axial data (see Bastiaansen \& Knosche, 2000, for a direct comparison).

\section{ERF Analysis}

For the computation of the event-related fields (ERFs), the MEG data were first low-pass filtered at $30 \mathrm{~Hz}$. Artifact-free epochs ranging from $150 \mathrm{msec}$ before onset of the CW to 
1000 msec after this point were averaged separately for each of the three conditions. A baseline correction between -150 msec and 0 was applied.

The significance of the difference between the conditions was evaluated by means of a cluster-based random permutation test (see Statistical Analyses).

\section{Time-Frequency Analysis of Power}

\section{Computation of Time-Frequency Representations}

In order to reveal event-related changes in power for the different frequency components of the MEG, timefrequency representations (TFRs) of the single trial data were computed by using the multitaper approach described by Mitra and Pesaran (1999). In order to optimize the tradeoff between time and frequency resolution, TFRs were constructed in two different, partially overlapping frequency ranges (see, e.g., Womelsdorf, Fries, Mitra, \& Desimone, 2006 for a similar approach to multitaper analysis). In the low-frequency range $(2-36 \mathrm{~Hz}), 2-\mathrm{Hz}$ frequency-smoothing and $500 \mathrm{msec}$ time-smoothing windows were used to compute power changes in frequency steps of $2 \mathrm{~Hz}$ and time steps of $10 \mathrm{msec}$. Effectively, in terms of temporal resolution, this means that any given time point in the resulting TFR is a weighted average of the time points ranging from $250 \mathrm{msec}$ before to $250 \mathrm{msec}$ after this time point. In the high-frequency range (30$100 \mathrm{~Hz}$ ), power changes were computed in 5-Hz frequency steps and 10-msec time steps, with a $10-\mathrm{Hz}$ frequencysmoothing and a $200 \mathrm{msec}$ time-smoothing (i.e., an interval of -100 to $100 \mathrm{msec}$ around each time point in the resulting TFR).

\section{Power Changes Time-locked to the Critical Word}

In order to investigate the local effects (i.e., right after the presentation of the $\mathrm{CW}$ ) of a syntactic violation on the TF data, we time-locked the single-trial TFRs to the CW. Then, TFRs were averaged for each subject, in a time range from $500 \mathrm{msec}$ before onset of the CW to $2500 \mathrm{msec}$ after this point, separately for each of the three conditions. The resulting subject-averaged power changes were expressed as a relative change from the reference interval, which ranged from 500 to $150 \mathrm{msec}$ before onset of the CW (because of the temporal smoothing involved in multitaper analysis, this reference interval ended $150 \mathrm{msec}$ before stimulus onset). This resulted in an ERD/ERS-like measure of relative power change (Pfurtscheller \& Lopes da Silva, 1999a; Pfurtscheller \& Aranibar, 1977). The significance of the difference between conditions was evaluated by means of a cluster-based random permutation test (see Statistical Analyses).

\section{Power Changes Time-locked to Sentence Onset}

In addition to the power changes that occur right after the $\mathrm{CW}$, we were also interested in the evolution of power changes over the entire time course of the sentence. Therefore, in a second analysis, we time-locked the single-trial TF presentations to the onset of the first word in each sentence. Although this has the disadvantage of resulting in a less precise time-locking with respect to the CW, it does allow for an evaluation of the power changes across the entire sentence, compared to a pre-sentence baseline. Thus, single-trial TFRs were averaged for each subject, in a time range from $500 \mathrm{msec}$ before onset of the first word in the sentence, to $1500 \mathrm{msec}$ after onset of the last word. Note that, because of the variable sentence length, the trials thus defined varied in length between 5000 msec (for 6-word sentences) and 7400 msec (for 10-word sentences). Subject averages were expressed as a relative change from the reference interval, which ranged from 500 to $150 \mathrm{msec}$ before the onset of the sentence-initial word. Statistical comparisons (see Statistical Analyses) were made between COR and CAT, between COR and RDM, and between CAT and RDM.

\section{Statistical Analyses}

The statistical significance of the differences between conditions, both for the observed ERFs and for the TFRs of power change, was evaluated by a cluster-based random permutation approach (see Maris \& Oostenveld, 2007 for details on the method). We used this statistical approach mainly because of its elegant handling of the multiple-comparisons problem: Because we have little a priori knowledge about when and where to expect condition differences (and as a result we cannot preselect time or frequency windows, nor MEG channels for statistical analysis), we would need to perform some $151 \times$ $18 \times 651$ (see below for an account of these figures) $=$ roughly 1.7 million statistical tests. The approach naturally takes care of interactions between channels, time points, and frequency bins by identifying clusters of significant differences between conditions in the time, space, and frequency dimensions, and effectively controls the Type 1 error rate in a situation involving multiple comparisons. The procedure is briefly described here (for an elaborate description of the approach, see Maris \& Oostenveld, 2007).

First, for every data point (sensor-time points for ERF data, sensor-TF points for TF data) a simple dependentsamples $t$ test is performed (giving uncorrected $p$ values). All data points that do not exceed a preset significance level (here 5\%) are zeroed. Clusters of adjacent non-zero data points are computed, and for each cluster a clusterlevel test statistic is calculated by taking the sum of all the individual $t$ statistics within that cluster.

Next, a null distribution is created as follows. Subject averages are randomly assigned to one of the two conditions 500 times, and for each of these randomizations, cluster-level statistics are computed. For each randomization, the largest cluster-level statistic is entered into the null distribution. Finally, the actually observed cluster-level test 


\section{ERF analysis}

\section{A ERF time courses}

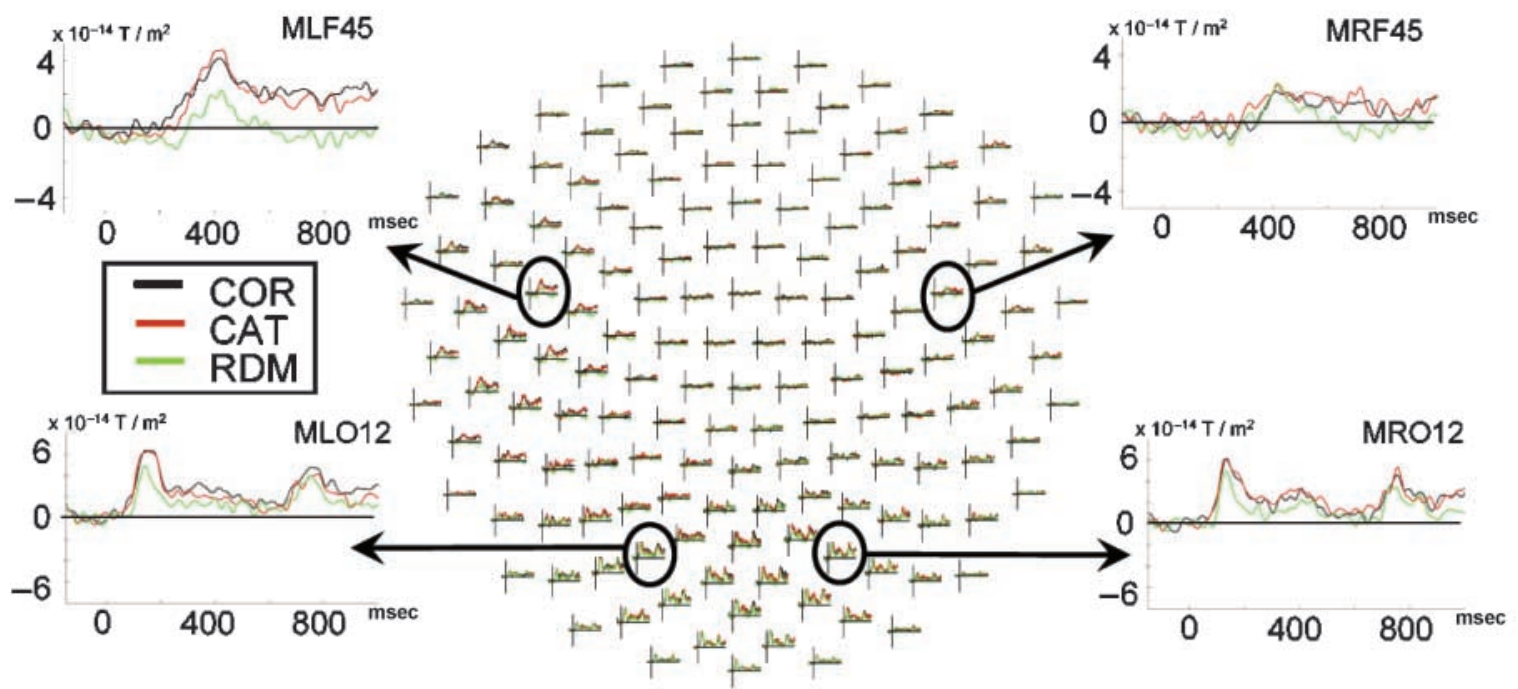

\section{B ERF scalp topographies}

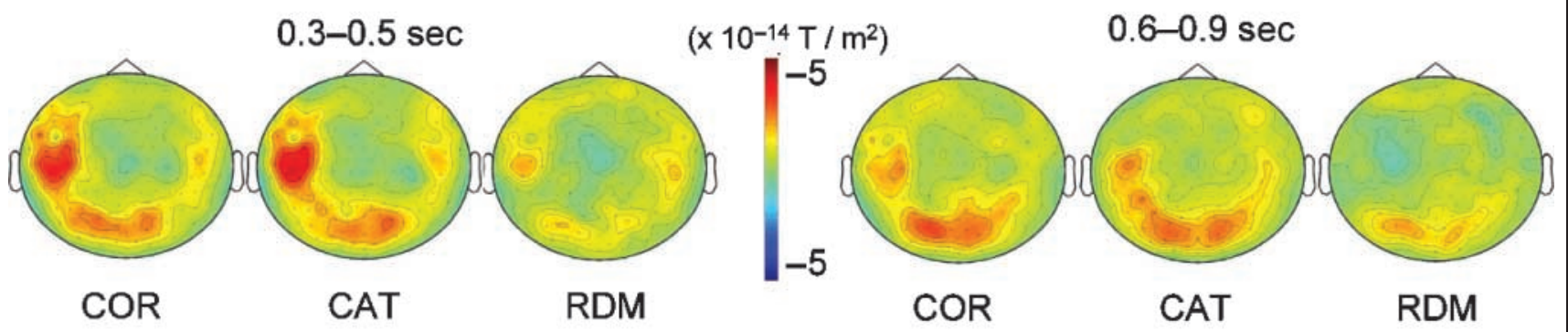

C ERF statistical analysis

Difference topographies $(0.3-0.5 \mathrm{sec})$

COR vs. RDM
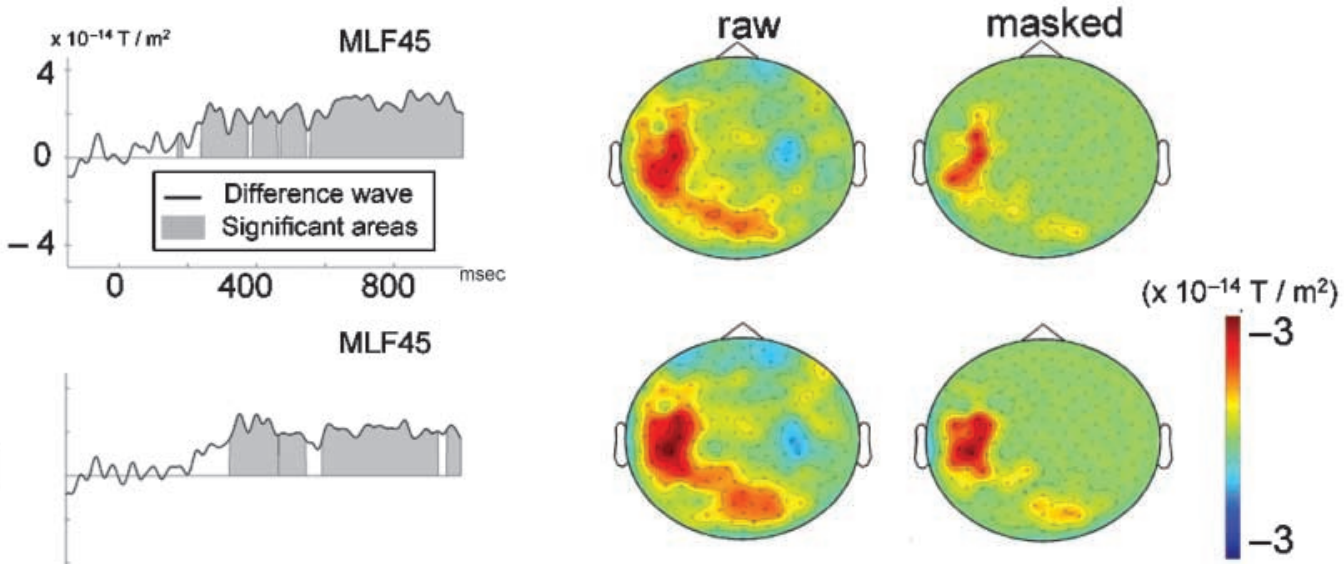

Figure 1. Results of the ERF analysis. (A) ERF time course at all channels, with insets for four representative sensor positions. (B) Scalp topographies of the ERFs for each of the three conditions, in two time windows. (C) Results of the statistical analysis of the ERF data. Gray areas under the curves in the left-hand plots indicate time regions where the contrasts show significant differences. In the right-hand plots, topographies show the raw difference between conditions for the two contrasts (raw), and the statistically thresholded difference (masked), where channels that do not differ significantly between the conditions are zeroed. 
Figure 2. TFRs showing the alpha-band difference between the COR and CAT conditions. TFRs are shown separately for the COR and CAT conditions, the raw difference between these conditions (COR-CAT), and the statistically thresholded difference (masked). $t=0$ corresponds to the onset of the critical word. The black rectangle in each TFR indicates the frequency range that is of interest in this figure. On the right-hand side, the raw and masked (see legend to Figure 1) topographies of the difference between COR and CAT are given.

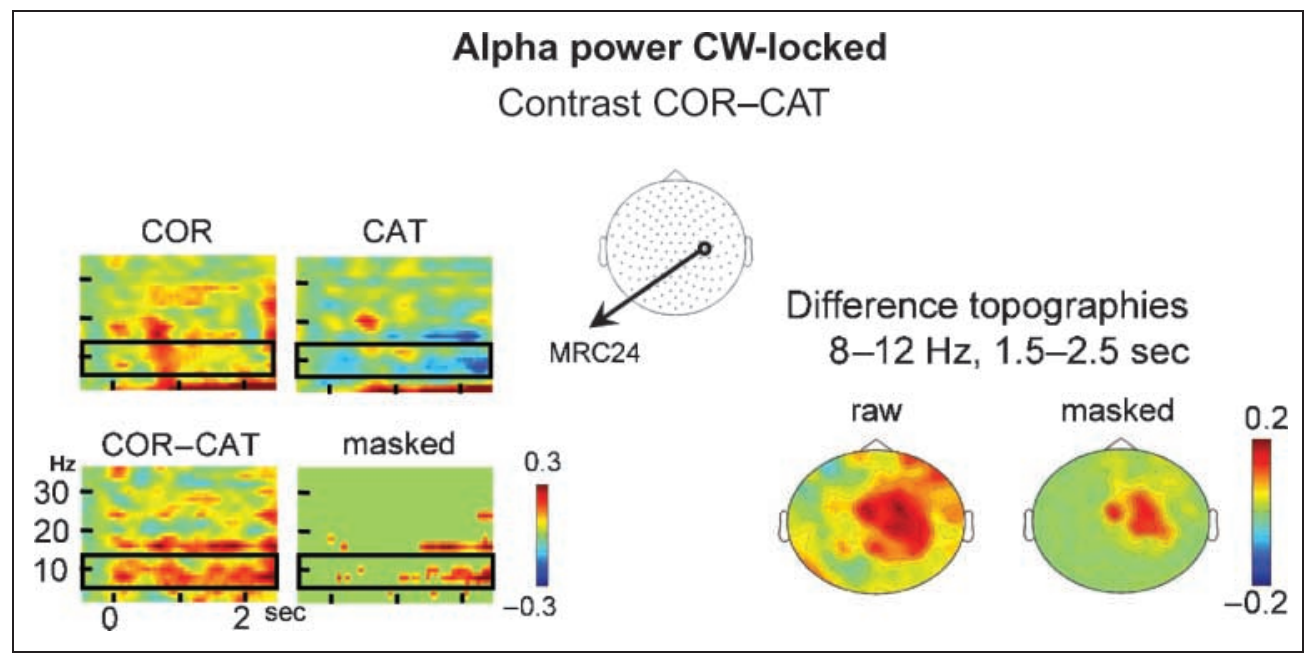

statistics are compared against the null distribution, and clusters falling in the highest or lowest 2.5 th percentile are considered significant.

This procedure only allows for pairwise comparisons. Therefore, for each of the three dependent measures (the ERFs, the TFRs time-locked to CW, and the TFRs time-locked to sentence onset), a set of three pairwise comparisons was performed: COR versus CAT, COR versus RDM, and CAT versus RDM.

For the ERF data, all 151 MEG channels and 1380 time points (corresponding to an interval of $150 \mathrm{msec}$ before to 1000 msec after CW onset, at $1200 \mathrm{~Hz}$ sampling rate) were entered into the analysis. For the CW-locked TF data, all 151 MEG channels, 301 time points ( -500 to 2500 around CW onset in 10-msec time steps), and 18 or 15 frequency bins $(2-36 \mathrm{~Hz}$ in $2-\mathrm{Hz}$ steps for the lowfrequency analysis and $30-100 \mathrm{~Hz}$ in $5-\mathrm{Hz}$ steps for the high-frequency analysis, respectively) were entered into the analysis. For the sentence-onset-locked TF data, all 151 MEG channels, 651 time points ( -500 to $6000 \mathrm{msec}$ after onset of the sentence-initial word, in 10-msec time steps), and 18 or 15 frequency bins (2-36 Hz in 2-Hz steps for the low-frequency analysis and 30-100 Hz in 5-Hz steps for the high-frequency analysis, respectively) were entered into the analysis.

\section{RESULTS \\ ERF Data}

Grand-average ERFs for the three conditions are presented in Figure 1. An early occipital deflection (around 100150 msec after CW onset) and a later bilateral, lefthemisphere-dominant temporal deflection (around 400 msec after CW onset) are present in all conditions. The scalp distribution of the latter effect is compatible with previous reports on the magnetic equivalent of the N400 (the N400m; e.g., Halgren et al., 2002; Helenius, Salmelin, Richardson, Leinonen, \& Lyytinen, 2002; Helenius, Salmelin, Service, \& Connolly, 1998; Salmelin, Service,
Kiesila, Uutela, \& Salonen, 1996). Starting from approximately 300 msec after CW onset, amplitudes for the RDM condition are significantly smaller than in the other two conditions over left temporal recording sites. This effect increases monotonically across time (Figure 1C), lasts until the end of the analyzed interval (which is $1000 \mathrm{msec}$ after CW onset, and is topographically stable across time; Figure $1 \mathrm{~B}$ and $\mathrm{C}$ ). In the statistical comparisons, this effect is expressed by a significant cluster both in the contrast COR-RDM $(p<.01)$ and in the contrast CAT-RDM ( $p<.01$; cf. Figure 1C). No significant differences were observed between the COR and CAT conditions.

\section{Power Changes Time-locked to the Critical Word}

For the low frequencies $(2-36 \mathrm{~Hz})$, the contrast COR-CAT yielded one significant cluster $(p<.001)$ showing effects both in the alpha (8-12 Hz) and in the lower-beta (13$18 \mathrm{~Hz}$ ) frequency ranges. The contrasts COR-RDM and CAT-RDM both revealed one significant cluster (both $p$ values $<0.001$ ) with effects in the lower-beta frequency range (13-18 Hz) only. For the high frequencies (30$100 \mathrm{~Hz}$ ), the contrast COR-CAT revealed one significant cluster $(p<.02)$ showing an effect in the high gamma frequency range $(60-80 \mathrm{~Hz})$. Below, we present the different effects for each frequency band in more detail. Note that because we were mainly interested in between-condition differences, we did not explicitly test whether power changes in the post-CW interval differed significantly from the pre-CW interval within each condition. We therefore only discuss within-condition power changes at a descriptive level.

\section{Alpha Band}

In the alpha frequency range (8-12 Hz; see Figure 2), correct sentences induced a small power increase in the first 1000 msec after CW onset relative to the pre-CW baseline, 
Figure 3. Effects in the lower-beta frequency range in the critical word-locked analysis. (A) TFRs are shown at three representative sensors, for each of the three conditions. The black rectangle in each TFR indicates the frequency range that is of interest in this figure. (B) The left-hand plot shows the contrast between COR and CAT sentences; raw and statistically thresholded difference TFRs are displayed for left and right frontal sensors. The right-hand plot gives the corresponding scalp topographies for two different time intervals, an early interval (0-600 msec) where a left frontal effect is observed, and a late interval (1000-2500 msec) where a mid-frontal to right frontal effect is observed. (C) Same as B, but for the COR-RDM contrast. (D) same as B, but for the CAT-RDM contrast.

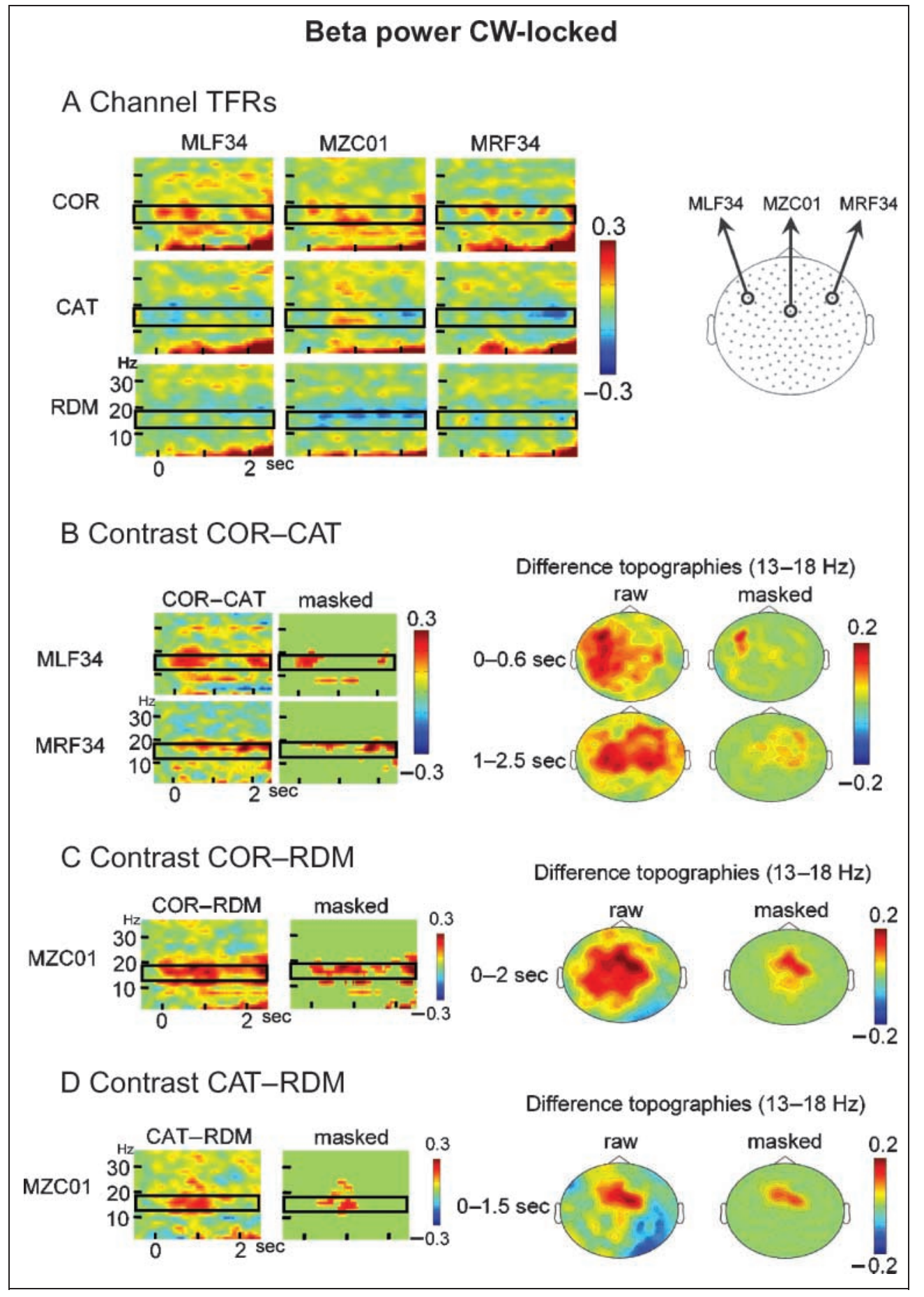

whereas word category violations induced a long-lasting (from 0 to $2500 \mathrm{msec}$ after $\mathrm{CW}$ ) power decrease. The difference was significant over midline and right-hemisphere frontal and central areas. The contrasts COR-RDM and CAT-RDM did not yield significant differences in this frequency range.

\section{Beta Band}

In the lower-beta frequency range (13-18 Hz; see Figure 3), COR sentences showed a long-lasting (0-2500 msec after
CW onset) power increase over frontal areas, whereas CAT sentences showed hardly any beta power changes, and RDM sentences showed a mid-frontal power decrease (see Figure 3A). In the COR-CAT contrast, larger power for COR sentences was observed over left frontal areas initially (0-600 msec after CW onset) and over right frontal areas subsequently (1000-2500 msec after CW onset; Figure 3B). The contrasts COR-RDM (Figure 3C) and CAT-RDM (Figure 3D) showed larger mid-frontal beta power for the COR and the CAT conditions, respectively, probably as a result of the drop in power in the RDM condition. 


\section{Gamma Band}

In the gamma frequency range (60-80 Hz; see Figure 4), a small increase in power was observed for the COR condition in the first 1000 msec after CW onset, whereas a small decrease in power was observed for the CAT condition in the same time interval. Significant gamma power differences between the two conditions were restricted to right temporal areas. The contrasts COR-RDM and CAT-RDM did not yield significant differences in this frequency range.

\section{Power Changes Time-locked to Sentence Onset}

The analyses of power changes across the entire sentence revealed significant differences in the contrast COR-CAT $(p<.03)$ and in the contrast COR-RDM $(p<.003)$. Both clusters showed effects in the theta band ( $4-8 \mathrm{~Hz})$ and in the lower-beta band (13-18 Hz). In the analyses of the high-frequency range (30-100 Hz), no differences were observed between the three conditions in any of the contrasts.

\section{Theta Band}

In the theta band, power was larger in the COR condition than in the CAT and RDM conditions, in a time interval from 3500 to 5000 msec for the COR-CAT contrast, and 3000$6000 \mathrm{msec}$ for the COR-RDM contrast, after onset of the sentence-initial word (see Figure $5 \mathrm{~A}-\mathrm{C}$ ). The difference is restricted to right centro-parietal areas in both contrasts. No power differences were observed between CAT and RDM.

In addition, a visual inspection of the time course of theta-band power in the three conditions (Figure 5D) suggests a linear increase in power across the sentence. We therefore performed a post hoc analysis in order to test for the significance of such a linear trend. To this end, a time interval from 0 to 4800 msec after sentence onset was selected. This interval corresponds to the presentation of the first eight words in the sentence, and was chosen because the amount of sentences containing more than eight words was relatively small, which may lead to more inaccurate power estimates after $4800 \mathrm{msec}$, due to a reduced signal-to-noise ratio (less trials in the subject averages). Then the 10 sensors showing maximal power across the three conditions were selected, and their time courses were averaged (see Figure 5D). Next, for each subject, a linear regression line was fitted through the individual theta power time course. Finally, for each condition, a one-sample $t$ test was performed on the individual beta weights (i.e., the slopes) of the resulting regression parameters, evaluating whether the beta weights differed significantly from zero on average. The results indicate the presence of a linear trend for COR $[t(17)=3.11, p=$ $.006]$, CAT $[t(17)=2.29, p=.035]$, but the linear trend did not reach significance for $\operatorname{RDM}[t(17)=2.01, p=$ .060]. Furthermore, despite the fact that toward the end of the analyzed interval the linear trends for CAT and RDM seem to deviate from the one for the COR condition, paired-sample $t$ tests showed that the slope of the linear trends did not differ for any of the possible pairwise contrasts (all $p$ values $>0.3$ ).

\section{Beta Band}

In the lower-beta range (13-18 Hz), power was larger for COR than for CAT and RDM in a time interval from roughly 2000 to $6000 \mathrm{msec}$ after sentence onset (Figure 6A-C). As can be seen in Figure 6A, this difference is due to an increase in beta power for COR, which is absent for CAT and RDM. The effect is present over mid-frontal areas as well as over left and right parietal areas, although in the COR-CAT contrast the left parietal area does not reach significance (see the topographies in Figure 6B and C).

Again, a visual inspection of the data suggested the presence of a linear trend, at least in the COR condition (Figure 6D). We therefore tested for the presence of such a linear trend in the same way as for the theta band. This analysis showed that for the COR condition this trend reaches significance $[t(17)=2.42, p=.027]$. Interestingly, in the CAT condition, beta power initially appears to linearly increase just as in the COR condition. This increase drops back to baseline in the time window in which the
Figure 4. TFRs showing the gamma-band difference between the COR and CAT conditions (see legend to Figure 2 for details).

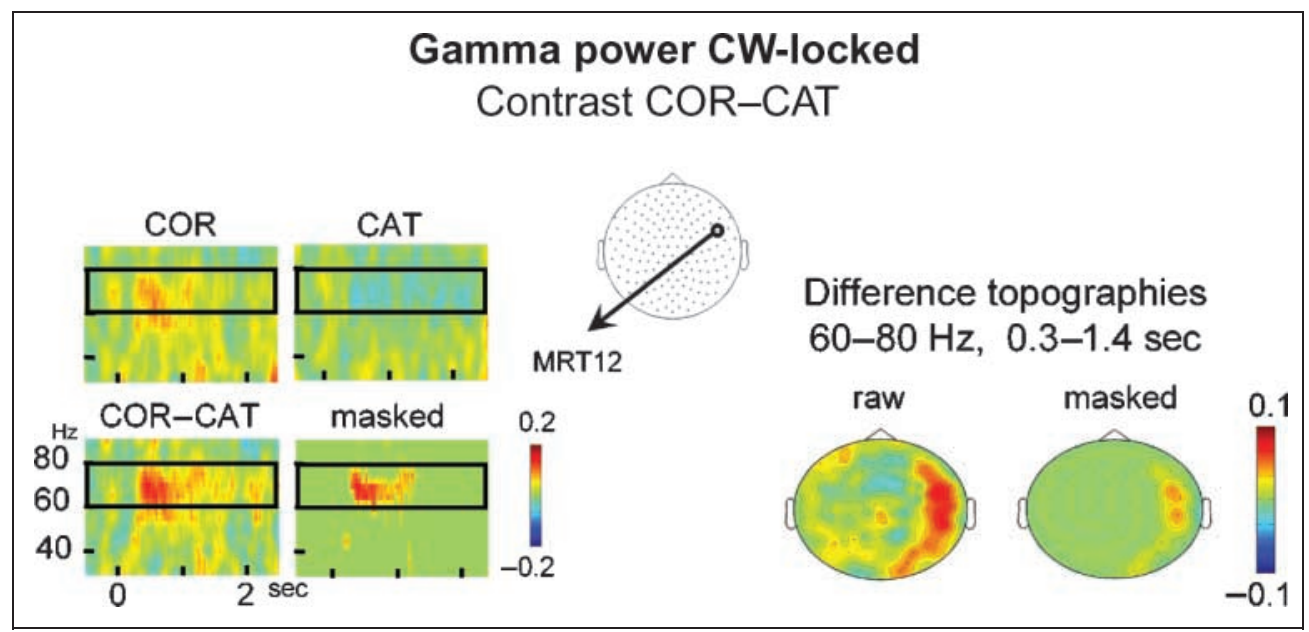


word category violation was presented (Figure 6D). Therefore, the linear trend for the CAT condition failed to reach significance $[t(17)=1.77, p=.095]$. For the RDM condition, no linear trend was present at all $[t(17)=-1.37, p=$ .189]. Pairwise $t$ testing for a slope difference between conditions indicated that the slope was largest for the COR condition [COR-CAT contrast: $t(17)=4.01, p=$ .001; COR-RDM contrast: $t(17)=3.42, p=.003]$ and similar for the CAT and RDM conditions [CAT-RDM contrast: $t(17)=0.31, p=.76]$.

\section{DISCUSSION}

We studied the oscillatory neuronal correlates of syntactic unification operations during on-line sentence compre- hension by recording MEG from subjects while they read syntactically correct sentences (COR), sentences containing a word category violation (CAT), and sentences in which the order of words was pseudorandomized in order to remove any syntactic structure (RDM). ERFs evoked by the CWs were compared between conditions. We were most interested, however, in the comparison of time-resolved power changes between conditions, both across the entire sentence, and as a result of CW presentation.

\section{ERF Data}

The CWs elicited, in all three conditions, a deflection peaking at 400 msec after CW onset, with a bilateral, leftdominant temporal scalp distribution. This ERF component is reminiscent of the magnetic equivalent of the
Figure 5. Effects in the theta frequency range, for the sentence-onset-locked analysis. (A) TFRs at one representative channel, for the three conditions separately. $t=0$ corresponds to the onset of the sentence-initial word. The black rectangle in each TFR indicates the frequency range that is of interest in this figure. (B) The left-hand plots show difference TFRs and statistically thresholded TFRs for the contrast between COR and CAT sentences. The right-hand plots show raw and statistically thresholded scalp topographies of the theta effect for the COR-CAT contrast. (C) Same as $\mathrm{B}$, but for the COR-RDM contrast. (D) Time course (average of 10 channels) of the evolution of theta power (4-7 Hz) across the entire sentence, for the three conditions. Yellow bars at the top of the graph indicate the timing of the presentation of the individual words in each sentence. Red areas in these bars indicate the proportion of word category violations occurring at the corresponding word position (CAT condition only). The height of the bars shows the proportion of sentences that still contain words at the corresponding word position. The transparent gray rectangle indicates the time window in which a word category violation could occur (CAT condition only). Note the slow increase in theta power across the sentence for all conditions.

\section{Theta power onset-locked}

\section{A Channel TFRs}

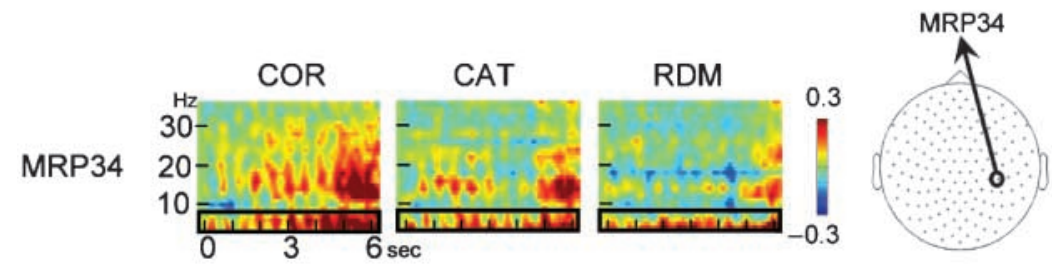

\section{B Contrast COR-CAT}

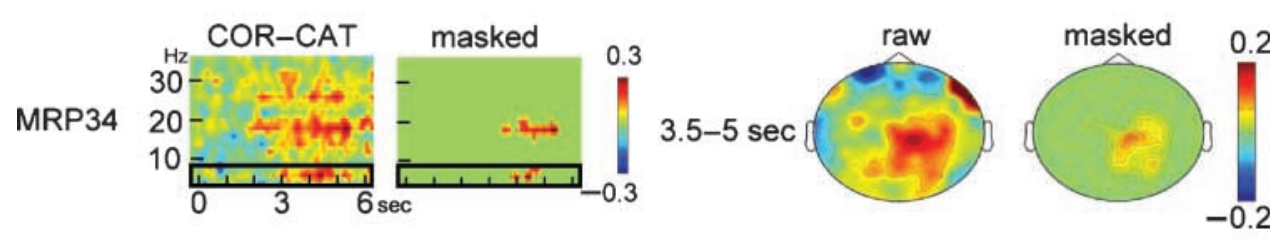

\section{Contrast COR-RDM}

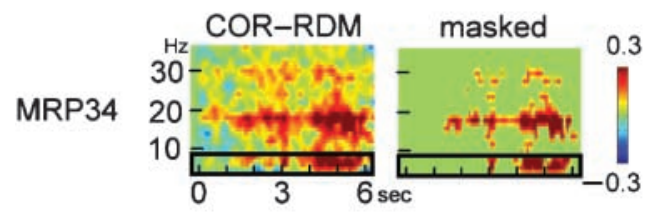

\section{Difference topographies $(4-8 \mathrm{~Hz})$}

$3-6 \mathrm{sec}$
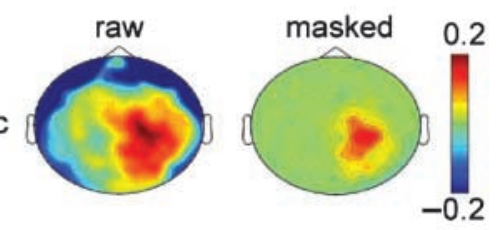

\section{Theta power time course}

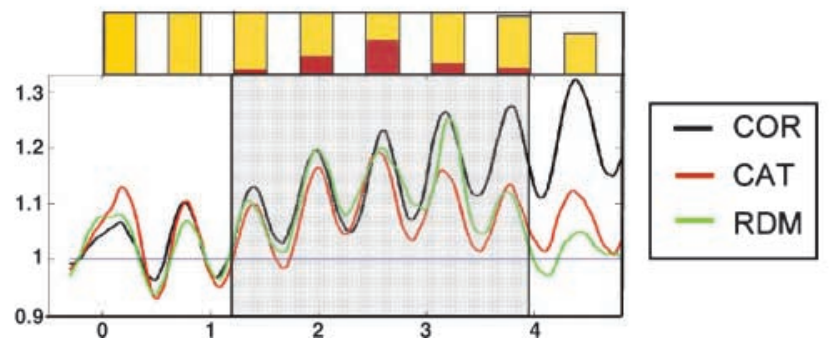


Figure 6. Effects in the lower-beta frequency range, for the sentence-onset-locked analysis (see legend to Figure 5 for details)

\section{Beta power onset-locked}

\section{A Channel TFRs}
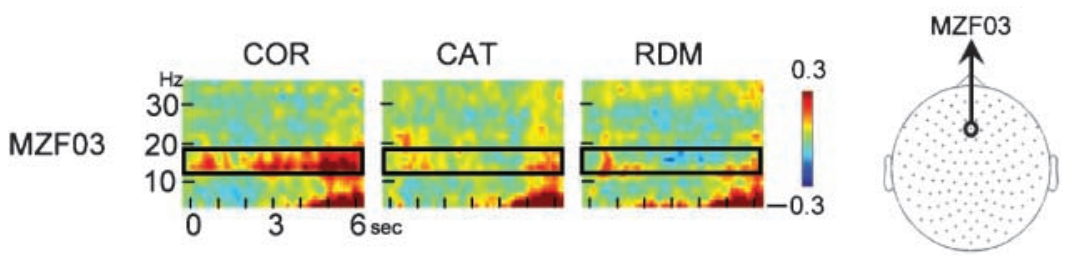

\section{B Contrast COR-CAT}

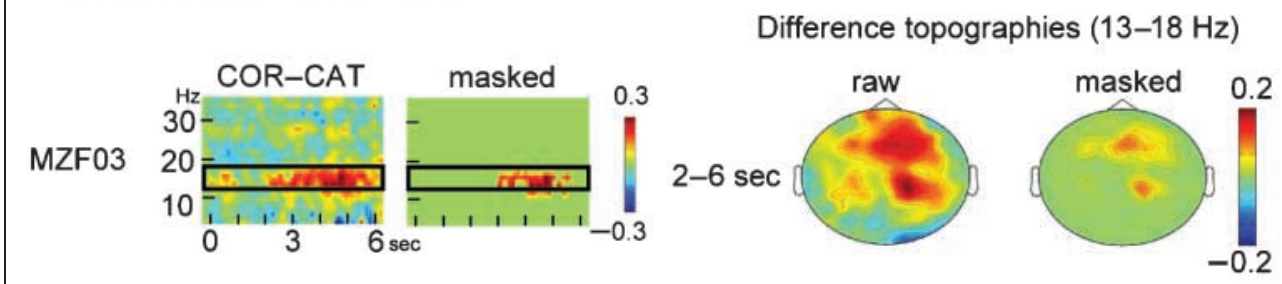

\section{Contrast COR-RDM}

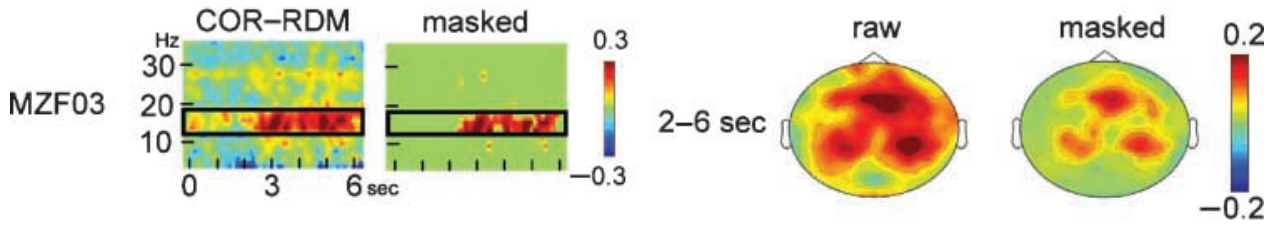

\section{Beta power time course}

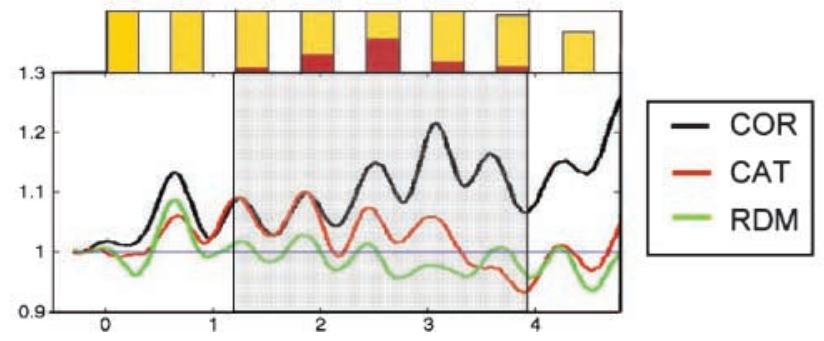

N400 (the N400m; e.g., Halgren et al., 2002; Helenius et al., 1998, 2002; Salmelin et al., 1996).

In addition to this component, we found a long-lasting (200-1000 msec after CW onset) ERF difference between sentences containing a syntactic structure (COR and CAT) on the one hand, and those without a syntactic structure (RDM) on the other hand. The topography of this difference, with a dominant left temporal focus and a quite weaker mid-occipital focus, was highly stable across the entire analyzed time interval (200-1000 msec after CW onset). Its stable topography, together with the fact that it increased monotonically throughout the post-CW interval, strongly suggests that this component is independent from the N400m component we observed in the data. The effect may be related to syntactic processing (because of the sensitivity to the syntactic manipulations), but given the pattern of results, it could also be related to semantic processing. An argument in favor of an interpretation of this effect in terms of semantic processing would be that it is only absent in the RDM condition, where the syntactic structure is so severely violated that semantic unification becomes impossible. However, for the following reasons, we have a (slight) preference for interpreting the effect in terms of syntactic processing. First, the effect consisted of a larger deflection for sentences containing a syntactic structure (even though it was violated in the CAT condition) than for sentences that were completely devoid of syntactic structure. Second, the topography of the effect is compatible with other MEG studies that have reported an involvement of left temporal cortex in syntactic processing (Kwon et al., 2005; Herrmann et al., 2003; Shtyrov, Pulvermuller, Näätänen, \& Ilmoniemi, 2003; Friederici, 
Wang, Herrmann, Maess, \& Oertel, 2000; Knosche, Maess, \& Friederici, 1999). Note that in these studies larger left temporal responses were found following syntactic violations (which are considered to increase syntactic processing) as compared to normal sentences. Although our random word order sentences also consisted of syntactic violations, one can assume that, in this case, the syntactic structure is so severely violated that syntactic processing is abandoned altogether. Thus, we consider the larger left temporal activity in the COR and CAT conditions as compared to the RDM conditions to be compatible with the larger activity commonly reported in that area after a single syntactic violation. Note, however, that we did not observe the latter effect in the CAT versus COR contrast.

If one is willing, for now, to go along with a syntactic interpretation of the larger left temporal deflections for COR and CAT versus RDM, one could raise the question of what aspect of syntactic processing is reflected by this effect. Lexico-syntactic retrieval (i.e., the retrieval of wordlevel syntactic information) is not a likely candidate because one would assume that lexico-syntactic information is retrieved for each word, even in the absence of sentential syntactic structure (as in the present RDM condition). Rather, we envisage two viable interpretations of the effect. First, it may be related to keeping lexico-syntactic information "on-line," so that this information is available for the neural structures involved in syntactic unification (e.g., left inferior frontal cortex). This account would be compatible with the MUC framework for language comprehension proposed by Hagoort (2005). A second candidate process is syntactic unification proper, although this would postulate a central role for left temporal cortex in syntactic unification, which is at odds both with findings from the neuroimaging literature (see, e.g., the review by Bookheimer, 2002) and with the MUC framework (Hagoort, 2005). The present results are inconclusive with respect to this issue.

Surprisingly, we did not find an ERF difference between the COR and CAT conditions. A word category violation, as in the CAT condition, is expected to yield a (magnetic equivalent) of a LAN or P600 component. In the current dataset, a small difference in ERF is, indeed, observed between COR and CAT in the P600 time interval (see, e.g., sensor MLF45 in Figure 1), but this difference did not reach statistical significance $(p>$.1). It is difficult to account for this lack of significance. It may be that the violation used here was too mild to induce a reliable magnetic equivalent of the LAN or P600.

\section{Time-Frequency Data}

The analysis of the event-related power changes revealed three types of effects: (1) an alpha and gamma decrease in the CAT versus the COR condition in the CW-locked analysis (Figures 2 and 4, respectively); (2) a graded effect in the lower-beta band in the CW-locked analysis, where larger power was associated with more intact syntactic structure $(\mathrm{COR}>\mathrm{CAT}>\mathrm{RDM})$, together with a linear increase across the COR sentences in that same frequency band in the sentence-onset-locked analysis (Figures 3 and 6 , respectively); and (3) a linear increase across the sentence in the theta band for the COR and CAT conditions (Figure 5).

Concerning the first type of effects, that is, the alpha and gamma power decreases after the word category violation, it should be noted that no differences in alpha and gamma were observed between syntactically structured (either COR or CAT) sentences on the one hand, and unstructured (RDM) sentences on the other hand. Because one would expect effects directly related to the processing of syntactic structure to yield differences between structured and unstructured sentences, it seems unlikely that the alpha and gamma effects are directly related to the analysis of the syntactic structure of a sentence. It rather seems that the effects are, in some way, related to the detection of a violation of a syntactically structured sentence.

As such, the gamma suppression observed after the word category violation is rather similar to the gamma suppression that we have reported in response to semantic violations (e.g., Hald et al., 2006; Hagoort et al., 2004), both in terms of reactivity (gamma increase in correct sentences, absence of gamma increase following semantic violations) and in terms of scalp distribution (right frontocentral in the EEG data by Hald et al., 2006; Hagoort et al., 2004; right centro-temporal in the present MEG data, which could both be explained with a tangential dipole in right temporal cortex pointing anteriorly). A tentative explanation of the drop in gamma after the word category violation would be to relate it to the semantic consequences of the syntactic violation-perhaps a temporary semantic unification failure-but this remains speculative.

The alpha-band effect, in turn, can be related to existing literature in two ways. It may be related to increased attention directed toward the violating word, which is compatible with many studies showing a clear relation between attentional processes and alpha power suppression (Bastiaansen, Bocker, \& Brunia, 2002; Klimesch, 1999; Foxe, Simpson, \& Ahlfors, 1998; Klimesch, Doppelmayr, Russegger, Pachinger, \& Schwaiger, 1998; Klimesch, Doppelmayr, Pachinger, \& Russegger, 1997; Brunia, 1993; Lopes da Silva, 1991; Steriade, Gloor, Llinás, Lopes da Silva, \& Mesulam, 1990). Alternatively, alpha-band effects have been related to semantic memory, as semantic judgment tasks have repeatedly been shown to induce alpha power decreases (see Rohm et al., 2001; Klimesch, Doppelmayr, Pachinger, \& Russegger, 1997; reviewed in Klimesch, 1999). Our present effect may be related to the effects found in these studies, although the exact nature of the relationship is as yet elusive.

The second type of effects appears to bear more directly on syntactic processing. In the lower-beta frequency range (13-18 Hz; Figures 3 and 6), power following the CW increased at frontal sensors for the correct sentences. After a word category violation, a sharply localized left frontal 
decrease was observed in an early (0-600 msec after CW onset) time interval, which later (after $1000 \mathrm{msec}$ ) shifted to a mid-frontal and right frontal decrease. However, in the analysis of power across the sentence, a linear increase was observed only in the COR condition, whereas in the CAT condition, an initial beta power increase was followed by a return to baseline during the time interval in which a violation occurred. This indicates that the power decrease (relative to the pre-CW baseline) observed in the CAT condition in the CW-locked analysis is, in fact, produced by the return to baseline of the gradual power increase across the sentence, as a result of encountering the syntactic violation. In addition, we did not observe any linear beta power increase for the sentences that were devoid of syntactic structure.

In sum, the beta power effects capture a process which increases in strength across a syntactically correct sentence, which is disrupted upon the occurrence of a syntactic violation, and which is not engaged during sentences without syntactic structure. Note that this pattern of results exactly fits the requirements for an effect to be linked to syntactic unification, as we noted in the Introduction. This makes oscillatory neuronal synchronization in the lower-beta frequency range a likely candidate mechanism for the neural implementation of syntactic unification operations. Note that the notion of lower-beta-band synchronization being involved in syntactic unification operations is also compatible with data reported by Weiss et al. (2005). These authors observed an increase in beta coherence between left frontal and left temporal electrodes after the presentation of syntactically complex object-relative clauses compared to the simpler subjectrelatives.

The third type of effects was found in the theta frequency band (here 4-8 Hz). There, we observed a gradual, linear increase in power across the COR and CAT sentences. The absence of theta-band effects in the CW-locked analyses further indicates that these effects are not very sensitive to syntactic violations. Therefore, the theta-band effect cannot be specifically related to the syntactic unification problems of the $\mathrm{CW}$ in the CAT condition. In a previous work we have related slow theta power changes across sentences to the gradual building up of a working memory trace of the language input (Bastiaansen et al., 2002a). The present theta-band results are largely compatible with such an interpretation (linear increases in COR and CAT, not in the hardly interpretable RDM sentences), although we feel that there are as yet too many degrees of freedom to be able to exclude possible alternative explanations. In addition, there are several problems with the interpretation of the current theta-band results in terms of WM increases. For instance, although it does not become apparent in the statistical analyses, the CAT condition shows a drop in power toward the end of the sentence (see Figure 5D). Furthermore, it is unclear why the RDM condition did not yield a significant trend (and shows the same drop in power as the CAT condition), as it is also likely to put high demands on WM. Taking everything into consideration, our explanation of the theta-band effects in terms of working memory is tentative at best.

The insensitivity of theta power to syntactic violations in the CW-locked analyses in this report stands in sharp contrast with our earlier findings of (CW-locked) frontal theta modulations after grammatical gender and number violations. However, these results were based on EEG rather than on MEG measurements, which tap into different subsets of the neural tissue (mainly gyral activity for EEG, and sulcal activity for MEG). We know from source localization studies of theta rhythms involved in domaingeneral error detection (Luu, Tucker, \& Makeig, 2004; Luu \& Tucker, 2001), as well as from simultaneous EEG/fMRI measurements (e.g., Scheeringa et al., 2008), that frontal theta originates from medial frontal cortex and/or anterior cingulate cortex. Increased neuronal activity in both these structures would lead to radially oriented dipoles that are invisible to MEG measurements (see, e.g., Stemmer, Vihla, \& Salmelin, 2004 for empirical evidence). Therefore, the frontal theta effects we reported elsewhere (Bastiaansen et al., 2002b) are not replicated in the present study.

The scalp topographies of the different effects vary widely and do not seem to be compatible with the lefthemisphere language network as identified with fMRI studies. It is difficult to explain the large variability in scalp topography of the effects at present, although it may be partially due to the fact that power changes quantify synchrony changes in widely distributed networks (which make sharp localizations of effects less meaningful anyhow).

One last caveat concerning the present data is that in all of the CW-locked analyses, there may have been substantial baseline differences between RDM on the one hand, and COR and CAT on the other. The reason for these differences is that the baseline in the CW-locked analyses coincides with the processing of the word preceding the CW. In the COR and CAT conditions, there is no difference in the stimulus materials up to this point. The RDM condition, in contrast, deviates from a natural language-processing situation and, as such, observed differences may stem from differences in extralinguistic factors such as attention or working memory. However, as the main points in this article are supported mostly by the sentence-onset-locked analyses, we do not consider this potential baseline problem to be of crucial importance.

In conclusion, we have shown that oscillatory neuronal activity in the lower-beta frequency range is likely related to the unification of the syntactic structure of a sentence, whereas synchronization changes across the sentence in the theta frequency range may possibly reflect the gradual emergence of a working memory trace as the sentence unfolds. Together with our earlier results, which suggest a relationship between semantic unification and gamma-band synchronization (Hald et al., 2006; Hagoort 
et al., 2004), a picture is starting to emerge in which functional networks are coupled through theta-band synchronization to establish a working memory trace of the linguistic input, while at the same time, a network synchronizing at gamma frequencies deals with semantic unification operations, and a lower-beta-band synchronous network is involved in syntactic unification. Note that we certainly do not want to take the position that different frequency bands implement certain cognitive functions. It is obvious that beta-band oscillations and gamma-band oscillations have many functional correlates. However, in the specific case of language comprehension, we are faced with the conceptual problem that different types of information (syntactic and semantic) have to remain segregated in what we know from the fMRI literature to be largely overlapping neuronal networks. It is therefore conceivable that, in the particular case of the language network, information flow related to semantics and syntax is kept separate through frequency coding.

However, in sketching this picture, we necessarily compare across, and make inferences across, different experimental designs, different subject samples, different dependent measures (power vs. coherence changes) and imaging modalities (EEG vs. MEG). Therefore, the different results obtained in the different approaches need to be replicated in more comprehensive within-subject designs in order to assess their robustness and general validity.

Reprint requests should be sent to Marcel C. M. Bastiaansen, Max Planck Institute for Psycholinguistics, P.O. Box 310, $6500 \mathrm{AH}$ Nijmegen, the Netherlands, or via e-mail: marcel.bastiaansen@ fcdonders.ru.nl.

\section{REFERENCES}

Bastiaansen, M., Bocker, K. B., \& Brunia, C. H. (2002). ERD as an index of anticipatory attention? Effects of stimulus degradation. Psychophysiology, 39, 16-28.

Bastiaansen, M., \& Hagoort, P. (2003). Event-induced theta responses as a window on the dynamics of memory. Cortex, 39, 967-992.

Bastiaansen, M., \& Hagoort, P. (2006). Oscillatory brain dynamics during language comprehension. In W. Klimesch \& C. Neuper (Eds.), Event-related dynamics of brain oscillations (Vol. 159, pp. 182-196). Amsterdam: Elsevier.

Bastiaansen, M., \& Knosche, T. R. (2000). Tangential derivative mapping of axial MEG applied to event-related desynchronization research. Clinical Neurophysiology, 111, 1300-1305.

Bastiaansen, M., Oostenveld, R., Jensen, O., \& Hagoort, P. (2008). I see what you mean: Theta power increases are involved in the retrieval of lexical semantic information. Brain and Language, 106, 15-28.

Bastiaansen, M., Van Berkum, J. J., \& Hagoort, P. (2002a). Event-related theta power increases in the human EEG during online sentence processing. Neuroscience Letters, 323, 13-16.

Bastiaansen, M., Van Berkum, J. J., \& Hagoort, P. (2002b). Syntactic processing modulates the theta rhythm of the human EEG. Neuroimage, 17, 1479-1492.
Bastiaansen, M., Van der Linden, M., ter Keurs, M., Dijkstra, T., \& Hagoort, P. (2005). Theta responses are involved in lexico-semantic retrieval during language processing Journal of Cognitive Neuroscience, 17, 530-541.

Bookheimer, S. (2002). Functional MRI of language: New approaches to understanding the cortical organization of semantic processing. Annual Review of Neuroscience, 25, 151-188.

Braeutigam, S., Bailey, A. J., \& Swithenby, S. J. (2001). Phase-locked gamma band responses to semantic violation stimuli. Brain Research, Cognitive Brain Research, 10, 365-377.

Brunia, C. H. M. (1993). Waiting in readiness: Gating in attention and motor preparation. Psychophysiology, 30, 327-329.

Foxe, J. J., Simpson, G. V., \& Ahlfors, S. P. (1998). Parietooccipital $\sim 10 \mathrm{~Hz}$ activity reflects anticipatory state of visual attention mechanisms. NeuroReport, 9, 3929-3933.

Friederici, A. D., Wang, Y., Herrmann, C. S., Maess, B., \& Oertel, U. (2000). Localization of early syntactic processes in frontal and temporal cortical areas: A magnetoencephalographic study. Human Brain Mapping, 11, 1-11.

Fries, P. (2005). A mechanism for cognitive dynamics: Neuronal communication through neuronal coherence. Trends in Cognitive Sciences, 9, 474-480.

Gibson, E. (1998). Linguistic complexity: Locality of syntactic dependencies. Cognition, 68, 1-76.

Gray, C. M., Konig, P., Engel, A. K., \& Singer, W. (1989). Oscillatory responses in cat visual cortex exhibit inter-columnar synchronization which reflects global stimulus properties. Nature, 338, 334-337.

Hagoort, P. (2005). On Broca, brain, and binding: A new framework. Trends in Cognitive Sciences, 9, 416-423.

Hagoort, P., Hald, L., Bastiaansen, M., \& Petersson, K. M. (2004). Integration of word meaning and world knowledge in language comprehension. Science, 304, 438-441.

Hald, L. A., Bastiaansen, M. C., \& Hagoort, P. (2006). EEG theta and gamma responses to semantic violations in online sentence processing. Brain and Language, 96, 90-105.

Halgren, E., Dhond, R. P., Christensen, N., Van Petten, C., Marinkovic, K., Lewine, J. D., et al. (2002). N400-like magnetoencephalography responses modulated by semantic context, word frequency, and lexical class in sentences. Neuroimage, 17, 1101-1116.

Helenius, P., Salmelin, R., Richardson, U., Leinonen, S., \& Lyytinen, H. (2002). Abnormal auditory cortical activation in dyslexia $100 \mathrm{msec}$ after speech onset. Journal of Cognitive Neuroscience, 14, 603-617.

Helenius, P., Salmelin, R., Service, E., \& Connolly, J. F. (1998). Distinct time courses of word and context comprehension in the left temporal cortex. Brain, 121, 1133-1142.

Herrmann, C. S., Friederici, A. D., Oertel, U., Maess, B., Hahne, A., \& Alter, K. (2003). The brain generates its own sentence melody: A Gestalt phenomenon in speech perception. Brain and Language, 85, 396-401.

Hummel, J., \& Holyoak, K. (1997). Distributed Representations of Structure: A Theory of Analogical Access and Mapping. Psychological Review, 104, 427-266.

Huyck, C. R. (2006). Creating hierarchical categories using cell assemblies. Connection Science, 19, 1-24.

Indefrey, P. (2004). Hirnaktivierungen bei syntaktischer Sprachverarbeitung: Eine Meta-Analyse. In H. M. Müller \& G. Rickheit (Eds.), Neurokognition der Sprache (pp. 31-50). Tübingen: Stauffenburg Verlag. 
Indefrey, P., \& Cutler, A. (2005). Prelexical and lexical processing in listening. In M. Gazzaniga (Ed.), The cognitive neurosciences III (pp. 759-774). Cambridge, MA: MIT Press.

Jackendoff, R. (2002). Foundations of language: Brain, meaning, grammar, evolution. Oxford, UK: Oxford University Press.

Klimesch, W. (1999). EEG alpha and theta oscillations reflect cognitive and memory performance: A review and analysis. Brain Research Reviews, 29, 169-195.

Klimesch, W., Doppelmayr, M., Pachinger, T., \& Ripper, B. (1997). Brain oscillations and human memory: EEG correlates in the upper alpha and theta band. Neuroscience Letters, 238, 9-12.

Klimesch, W., Doppelmayr, M., Pachinger, T., \& Russegger, H. (1997). Event-related desynchronization in the alpha band and the processing of semantic information. Brain Research, Cognitive Brain Research, 6, 83-94.

Klimesch, W., Doppelmayr, M., Russegger, H., Pachinger, Th., \& Schwaiger, J. (1998). Induced alpha band power changes in the human EEG and attention. Neuroscience Letters, 244, 73-76

Knosche, T. R., Maess, B., \& Friederici, A. D. (1999). Processing of syntactic information monitored by brain surface current density mapping based on MEG. Brain Topography, 12, 75-87.

Konig, P., \& Schillen, T. B. (1991). Stimulus-dependent assembly formation of oscillatory responses: I. Synchronization. Neural Computation, 3, 155-166.

Kwon, H., Kuriki, S., Kim, J. M., Lee, Y. H., Kim, K., \& Nam, K. (2005). MEG study on neural activities associated with syntactic and semantic violations in spoken Korean sentences. Neuroscience Research, 51, 349-357.

Lopes da Silva, F. H. (1991). Neural mechanisms underlying brain waves: From neural membranes to networks. Electroencephalography and Clinical Neurophysiology, 79, 81-93.

Luu, P., \& Tucker, D. M. (2001). Regulating action: Alternating activation of midline frontal and motor cortical networks. Clinical Neurophysiology, 112, 1295-1306.

Luu, P., Tucker, D. M., \& Makeig, S. (2004). Frontal midline theta and the error-related negativity: Neurophysiological mechanisms of action regulation. Clinical Neurophysiology, 115, 1821-1835

Maris, E., \& Oostenveld, R. (2007). Nonparametric statistical testing of EEG- and MEG-data. Journal of Neuroscience Methods, 164, 177-190.

Mitra, P. P., \& Pesaran, B. (1999). Analysis of dynamic brain imaging data. Biophysical Journal, 76, 691-708.

Nieuwland, M. S., \& Van Berkum, J. J. (2006). When peanuts fall in love: N400 evidence for the power of discourse. Journal of Cognitive Neuroscience, 18, 1098-1111.

Pfurtscheller, G., \& Aranibar, A. (1977). Event-related cortical desynchronization detected by power measurements of scalp EEG. Electroencephalography and Clinical Neurophysiology, 42, 817-826.

Pfurtscheller, G., \& Berghold, A. (1989). Patterns of cortical activation during planning of voluntary movement. Electroencephalography and Clinical Neurophysiology, 72, 250-258.

Pfurtscheller, G., \& Lopes da Silva, F. H. (1999a). Eventrelated EEG-MEG synchronization and desynchronization: Basic principles. Clinical Neurophysiology, 110, 1842-1857.

Pfurtscheller, G., \& Lopes da Silva, F. H. (Eds.) (1999b). Event-related desynchronization: Handbook of electroencephalography and clinical neurophysiology, revised series (Vol. 6). Amsterdam: Elsevier.
Pfurtscheller, G., \& Neuper, C. (1997). Motor imagery activates primary sensorimotor area in humans. Neuroscience Letters, 239, 65-68.

Pulvermueller, F. (1999). Words in the brain's language. Behavioural Brain Sciences, 22, 253-336.

Pulvermueller, F. (2002). A brain perspective on language mechanisms: From discrete neuronal ensembles to serial order. Progress in Neurobiology, 67, 85-111.

Rohm, D., Klimesch, W., Haider, H., \& Doppelmayr, M. (2001). The role of theta and alpha oscillations for language comprehension in the human electroencephalogram. Neuroscience Letters, 310, 137-140.

Salmelin, R., \& Kujala, J. (2006). Neural representation of language: Activation versus long-range connectivity. Trends in Cognitive Sciences, 10, 519-525.

Salmelin, R., Service, E., Kiesila, P., Uutela, K., \& Salonen, O. (1996). Impaired visual word processing in dyslexia revealed with magnetoencephalography. Annals of Neurology, 40, 157-162.

Scheeringa, R., Bastiaansen, M. C. M., Petersson, K. M., Oostenveld, R., Norris, D. G., \& Hagoort, P. (2008). Frontal theta EEG activity correlates negatively with the default mode network in resting state [Special issue on simultaneous EEG/fMRI]. International Journal of Psychophysiology, 67, 242-251.

Shtyrov, Y., Pulvermuller, F., Näätänen, R., \& Ilmoniemi, R. J. (2003). Grammar processing outside the focus of attention: An MEG study. Journal of Cognitive Neuroscience, 15, 1195-1206.

Singer, W. (1993). Synchronization of cortical activity and its putative role in information processing and learning. Annual Review of Physiology, 55, 349-374.

Singer, W. (1999). Neuronal synchrony: A versatile code for the definition of relations? Neuron, 24, 49-65, $111-125$.

Stemmer, B., Vihla, M., \& Salmelin, R. (2004). Activation of the human sensorimotor cortex during error-related processing: A magnetoencephalography study. Neuroscience Letters, 362, 44-47.

Steriade, M., Gloor, P., Llinás, R. R., Lopes da Silva, F. H., \& Mesulam, M. M. (1990). Basic mechanisms of cerebral rhythmic activities. Electroencephalography and Clinical Neurophysiology, 76, 481-508.

Van Berkum, J. J., Koornneef, A. W., Otten, M., \& Nieuwland, M. S. (2007). Establishing reference in language comprehension: An electrophysiological perspective. Brain Research, 1146, 158-171.

Van Berkum, J. J., Zwitserlood, P., Bastiaansen, M., Brown, C., \& Hagoort, P. (2004). So who's "he" anyway? Differential ERP and ERSP effects of referential success, ambiguity and failure during spoken language comprehension. Paper presented at the Annual Meeting of the Cognitive Neuroscience Society, San Francisco.

Varela, F., Lachaux, J. P., Rodriguez, E., \& Martinerie, J. (2001). The brainweb: Phase synchronization and large-scale integration. Nature Reviews Neuroscience, 2, 229-239.

Weiss, S., \& Mueller, H. M. (2003). The contribution of EEG coherence to the investigation of language. Brain and Language, 85, 325-343.

Weiss, S., Mueller, H. M., Schack, B., King, J. W., Kutas, M., \& Rappelsberger, P. (2005). Increased neuronal communication accompanying sentence comprehension. International Journal of Psychophysiology, 57, 129-141.

Womelsdorf, T., Fries, P., Mitra, P. P., \& Desimone, R. (2006). Gamma-band synchronization in visual cortex predicts speed of change detection. Nature, 439, 733-736. 